Another issue is the very short-term nature of this study for a therapy that is probably going to have to continue for the lifetime of the patients. This has a major impact in the area of complications, as some caregivers may lose enthusiasm for ITB if significant complications continue or become additive over a number of years and the benefit of the therapy becomes less clear. The list of complications (most manageable and minor like constipation, but others more severe, like sudden baclofen withdrawal, catheter failure, and deep infections) are an ongoing concern as long as the child has an implanted pump. These issues do not end by 18 months. ${ }^{2}$
I would like to make an appeal for considering ITB as a long-term therapy which requires an approach like other long-term functional implants, such as total joint replacements. Over the 50 or 60 years that these patients may need ITB, I would strongly advocate the importance of establishing a registry in which specific data such as that reported here ${ }^{1}$ are collected on a defined schedule along with detailed data on complications and dosing over time. This approach would have a higher possibility of providing useful long-term efficacy and cost/benefit data then attempting a short-term double blinded controlled trial.

\title{
REFERENCES
}

1. Morton RE, Gray N, Vloeberghs M. Controlled study to monitor the effects of continuous intrathecal baclofen infu-

sion in non-ambulant children with cerebral palsy. Dev Med
Cbild Neurol 2011; 53: 736-41. DOI: 10.1111/j.14698749.2011 .
2. Borowski A, Littleton AG, Borkhuu B, et al. Complications of intrathecal baclofen pump therapy in pediatric patients. $\mathcal{F}$ Pediatr Orthop 2010; 30: 76-81.

\section{Effects of a home-based treadmill training exercise program on impairment and function}

\author{
SHERYL LOW \\ Department of Physical Therapy, California State University, Northridge, CA, USA.
}

doi: 10.1111/j.1469-8749.2011.04030.x

\section{This commentary is on the original article by Johnston et al. on pages 742-750 of this issue.}

Research in pediatrics is always a challenge: locating and recruiting enough participants, matching children in groups, and designing a study that is randomized with relevant interventions. The study by Johnston et al. ${ }^{1}$ accomplishes all these difficult tasks. This multi-site study is the first randomized controlled trial that compared a partial-body-weight supported treadmill training intervention and an exercise intervention. While the improvements between the two groups were not statistically significant, there were other reasons this research should be considered significant.

The authors compare two forms of exercise intervention in the home: a supported speed treadmill training exercise program (SSTTEP) and an individualized exercise program for children with spastic cerebral palsy (CP). Historically, treadmill training has occurred in the clinic. Many studies have investigated this intervention with adults, and only recently has it been investigated in pediatric rehabilitation. ${ }^{2}$ This study is unique in that it showed positive changes in gait speeds and the Pediatric Outcomes Data Instrument (PODCI) that were retained 4 weeks after the completion of a 12-week homebased treadmill training exercise program. The authors lay out a protocol for training parents to carry out a home exercise treadmill training program that is reproducible. In light of current healthcare funding, a home exercise treadmill training program may be a realistic option for improving function for children with CP.
Another interesting aspect of this research is the use of minimal clinically important difference (MCID) to measure change and to interpret the results. The use of MCID is a systematic method of converting effect size to MCID in the units of the tool used to measure change in the study. The use of MCID for this study is based on values established by Oeffinger et al. ${ }^{3}$ for ambulatory children with CP. Johnston et al. recognize that while changes may not be statistically significant, they can be clinically meaningful. In using the MCID as an additional way to interpret the data, they found that the SSTTEP group showed greater improvements using the PODCI and gait speed, and that the individualized exercise program group showed greater changes on the Gross Motor Function Measure. They also found that children younger than 10 made greater gains than older children using this intensive intervention. By using the MCID to further analyze the data, the authors are opening up new relevant questions to investigate for future research.

This research also informs the ongoing discussion surrounding the role of impairment-based interventions and activity-based interventions on functional improvements for children with CP. ${ }^{4}$ While the statistical results were nonsignificant, the SSTTEP group maintained changes 4 weeks after the end of the intervention while the individualized exercise program group did not. The authors suggest motor learning and retention as one possible explanation. Purposeful practice and repetition around a motor activity, integrated into daily activities, can organize the learning of a new skill allowing increased retention. ${ }^{5}$

What is the best therapeutic intervention to improve gait for children with CP? While this study does not answer that question, it provides a reproducible model of testing the question, and offers suggestions for building on the results of previous research. 
1. Johnston JE, Watson KE Ross SA, et al. Effects of a

supported speed treadmill training exercise program on impairment and function for children with cerebral palsy. Dev Med Child Neurol. 2011; 53: 742-50. DOI: 10.1111/j.14698749.2011.03990.x.

2. Damiano DL, DeJong SL. A systematic review of the effectiveness of treadmill training and body weight support in pediatric rehabilitation. 7 Neurol Phys Ther 2009; 33: 4. Damiano DL. Activity, activity, activity: rethinking our physi$27-44$.

3. Oeffinger D, Bagley A, Rogers S, et al. Outcome tools used for ambulatory children with cerebral palsy: responsiveness and 5 . minimum clinically important differences. Dev Med Child Neurol 2008; 50: 918-25. cal therapy approach to cerebral palsy. Phys Ther 2006; 86: 1534-40.

5. Valvano J, Rapport MJ. Activity-focused motor interventions for infants and young children with neurological conditions. Infants Young Cbild 2006; 19: 292-307.

\section{How do we use the assessment of general movements in clinical practice?}

\section{ALICIA SPITTLE \\ Murdoch Childrens Research Institute, University of Melbourne, Victoria, Australia.}

doi: 10.1111/j.1469-8749.2011.04034.x

This commentary is on the original article by Hamer et al. on pages 751-756 of this issue.

The development of movement is a multifaceted process that begins within the first trimester of pregnancy. General movements, which are characterized by their complex, variable, and fluent movement sequences, appear as early as 9 weeks post conception. ${ }^{1}$ Heinz Prechtl, a pioneer in the assessment of infants' movement, was the first to describe how general movements could provide information on the integrity of the young nervous system. His work, along with that of many others, has demonstrated that general movements are predictive of cerebral palsy and neurological impairments, especially when assessed during the 'fidgety' age between 9 and 16 weeks. ${ }^{2}$ More recently, Hadders-Algra et al. ${ }^{3}$ have used a different method to assess general movements at the fidgety age, in which abnormal general movements are further classified as mildly abnormal or definitely abnormal. The study by Hamer et al. explores the predictive validity of this method and whether it can be improved by the additional assessment of specific characteristics of movements. ${ }^{4}$ The study of 46 infants, at high risk of developmental problems due to perinatal events, investigates the predictive value of definitely abnormal general movements and other movements patterns at 9 to 12 weeks of age for a comprehensive range of neurodevelopmental outcomes including cerebral palsy.

The study has two important findings. The first is that the additional assessment of stiff movements (but not the asymmetric tonic neck reflex and cramped synchronized movements at fidgety age) improves the predictive validity for cerebral palsy and motor performance at 18 months. This assists clinicians in adding an extra observational tool to their basket of assessments at 3 months. The other key finding of this study is that whilst many others have previously reported that fidgety movements have excellent specificity for normal developmental outcome, ${ }^{2}$ the authors reported that four children with presence of fidgety movements went on to develop cerebral palsy. $^{4}$

Given the multitude of influences on development, it is unlikely that as clinicians and researchers we will ever be able to predict with absolutely certainty whether a child will go on to have cerebral palsy or another developmental impairment from one assessment. The authors suggest that general movements can be used as a diagnostic tool for prediction of cerebral palsy; however, their research and other studies ${ }^{2,5}$ would suggest it is more of a complementary assessment that can help with prediction of adverse neurodevelopmental outcome - but not diagnosis. Rather, the role of early assessment of general movements for clinical use is to ensure that those infants most at risk of developmental problems are identified and appropriately monitored, and where appropriate, early interventions commenced. Importantly, the assessment of general movements and the research of Hamer et al. demonstrates that children who have cerebral palsy have alterations in movement patterns even before a great deal of voluntary movement is present and it is not always appropriate to wait until a child is 2 years of age to screen for movement disorders. It is essential that tools such as general movements assessments are not used in isolation as a diagnostic tool, and that both clinicians and parents are aware of their predictive value, to ensure that families are counselled appropriately. It is also vital for research to tell us the predictive value of a test; however, in the clinical setting, we should use all findings available from the physical examination, interview with the family, medical history of the infant, and radiological studies (where available) when making decisions.

\section{REFERENCES}

1. Luchinger AB, Hadders-Algra M, van Kan CM, de Vries JI. Fetal onset of general movements. Pediatr Res 2008; 63: $191-5$

2. Einspieler C, Prechtl HF. Prechtl's assessment of general movements: a diagnostic tool for the functional assessment of the young nervous system. Ment Retard Dev Disabil Res Rev 2005; 11: 61-7.

3. Hadders-Algra M. General movements: a window for early identification of children at high risk for developmental disorders. F Pediatr 2004; 145:(Suppl. 2) S12-8.
4. Spittle AJ, Boyd RN, Inder TE, Doyle LW. Predicting motor development in very preterm infants at 12 months' corrected age: the role of qualitative magnetic resonance imaging and general movements assessments. Pediatrics 2009; 123: $512-7$. 